\title{
Dynamin-1-Like Protein Inhibition Drives Megamitochondria Formation as an Adaptive Response in Alcohol-Induced Hepatotoxicity
}

Elena Palma, ${ }^{* \dagger}$ Xiaowen Ma ${ }^{\ddagger}$ Antonio Riva, ${ }^{\dagger \dagger}$ Valeria Iansante, ${ }^{\S}$ Anil Dhawan, ${ }^{\S}$ Shaogui Wang, ${ }^{\ddagger}$ Hong-Min Ni, ${ }^{\ddagger}$ Hiromi Sesaki, Roger Williams, ${ }^{* \dagger}$ Wen-Xing Ding, ${ }^{\ddagger}$ and Shilpa Chokshi ${ }^{* \dagger}$

From Institute of Hepatology, ${ }^{*}$ Foundation for Liver Research, London, United Kingdom; the Faculty of Life Sciences and Medicine ${ }^{\dagger}$ and the Institute of Liver Studies, ${ }^{\S}$ King's College London, London, United Kingdom; the Department of Pharmacology, Toxicology and Therapeutics, ${ }^{\ddagger}$ University of Kansas Medical Center, Kansas City, Kansas; and the Department of Cell Biology, "Johns Hopkins University School of Medicine, Baltimore, Maryland

Accepted for publication

November 2, 2018.

Address correspondence to Shilpa Chokshi, Ph.D., Institute of Hepatology, 111 Coldharbour Ln, SE5 9NT London, United Kingdom. E-mail: s.chokshi@ researchinliver.org.uk.

\begin{abstract}
Despite the growing global burden of alcoholic liver diseases, therapeutic options are limited, and novel targets are urgently needed. Accumulating evidence suggests that mitochondria adapt in response to ethanol and formation of megamitochondria in the livers of patients is recognized as a hallmark of alcoholic liver diseases. The processes involved in ethanol-induced hepatic mitochondrial changes, the impact on mitochondria-shaping proteins, and the significance of megamitochondria formation remain unknown. In this study, we investigated the mitochondrial and cellular response to alcohol in hepatoma cell line VL-17A. The mitochondrial architecture rapidly changed after 3 or 14 days of ethanol exposure with double-pronged presentation of hyperfragmentation and megamitochondria, and cell growth was inhibited. Dynamin-1-like protein (Drp1) was identified as the main mediator driving these mitochondrial alterations, and its genetic inactivation was determined to foster megamitochondria development, preserving the capacity of the cells to grow despite alcohol toxicity. The role of Drp1 in mediating megamitochondria formation in mice with liver-specific inactivation of Drp1 was further confirmed. Finally, when these mice were fed with ethanol, the presentation of hepatic megamitochondria was exacerbated compared with wild type fed with the same diet. Ethanol-induced toxicity was also reduced. Our study demonstrates that megamitochondria formation is mediated by Drp1, and this phenomenon is a beneficial adaptive response during alcohol-induced hepatotoxicity. (Am J Pathol 2019, 189: 580-589; https://doi.org/10.1016/j.ajpath.2018.11.008)
\end{abstract}

Alcoholic liver disease (ALD) encompasses multiple clinical presentations, ranging from simple steatosis to steatohepatitis, fibrosis, and cirrhosis, and can also manifest as severe alcoholic hepatitis. The pathobiology of ALD is not fully elucidated, and this has led to a lack of treatment options for this disorder, which represents 1 of the 10 most common causes of death in the Western world. ${ }^{1}$

Mitochondria play an essential role within the complex disease processes associated with ALD not only as the central location for alcohol-metabolizing enzymes, but also as active mediators in the response to alcohol toxicity. ${ }^{2,3}$ In hepatocytes, ethanol oxidation perturbs the homeostasis of several mitochondrial pathways involved in glucose/lipid metabolism and energy conversion. Ethanol also dramatically increases oxidative stress, which directly drives changes in mitochondrial proteins, lipids, and mitochondrial DNA, affecting functionality and cellular viability. ${ }^{4}$ More important, the morphology and the functionality of mitochondria are strictly correlated, and mitochondrial dynamics, with cycles of fusion (binding of two organelles) and fission (mitochondrial fragmentation), are constantly adjusting mitochondrial shape to maintain a pool of fully operative organelles. The balance between mitochondrial fusion and

Supported by the Foundation for Liver Research (S.C.) and NIH grants R01 AA020518, U01 AA024733, R21 AA027250, P20GM103549, and P30GM118247 (W.-.X.D.).

Disclosures: None declared. 
fission determines the architecture of the mitochondrion, which is necessary for the preservation of cellular and tissue integrity. These processes regulate the selective removal of damaged organelles (mitophagy) through fission and the maintenance of the bioenergetic efficiency through fusion. ${ }^{5}$ Fusion and fission are driven primarily through the activity of multiple mitochondria-shaping proteins, which act together to maintain a balance between these two antagonistic events. ${ }^{6}$ When either process is blocked, the final morphology of the mitochondrion is the consequence of unopposed progression toward the other side of the equilibrium. Although new members of this family are continuing to be discovered, the best characterized include mitofusin- 1 and mitofusin-2, which localize on the outer mitochondrial membrane and are essential for mitochondrial tethering to initiate the fusion process. ${ }^{7}$ Conversely, dynamin-1-like protein (Drp1; gene: $D N M 1 L$ ) drives fragmentation; when activated, this cytosolic GTPase protein translocates to mitochondria and assembles in multimers to constrict and fragment the organelle. ${ }^{8-10}$ Several proteins localized on the outer mitochondrial membrane have been proposed as Drp1 receptors, including mitochondrial fission 1 protein, mitochondrial fission factor, and mitochondrial dynamics protein MID49 and mitochondrial dynamics protein MID51 ${ }^{11}$; however, their exact mechanisms of action and the effect on mitochondrial morphology are still under debate. ${ }^{12}$ Alterations in mitochondria-shaping proteins or in the equilibrium between fusion and fission have been associated with the pathogenesis of several neurodegenerative and metabolic disorders. ${ }^{13,14}$

The current study elucidates the impact of ethanol on mitochondrial morphology in the liver and identifies, for the first time, the main regulator involved in the morphologic changes induced by ethanol on hepatic mitochondria, opening new perspectives for potential therapeutic strategies in ALD.

\section{Materials and Methods}

\section{Cellular Models}

Human hepatoma VL-17A cell line, stably expressing alcohol dehydrogenase and cytochrome P450 2E1, ${ }^{15}$ was cultured in complete Dulbecco's modified Eagle's medium containing zeocin and geneticin at $400 \mu \mathrm{g} / \mathrm{mL}$ in the presence/absence of ethanol at $100 \mathrm{mmol} / \mathrm{L}$ for 3 or 14 days, and ethanol was replaced at every passage. Treated cells were kept in an incubator saturated with ethanol to minimize evaporation (open petri dish containing $200 \mathrm{mmol} / \mathrm{L}$ ethanol).

Three cell models were derived from VL-17A. The first model included VL-17A cells transiently transfected with mitochondrially targeted red fluorescent protein (RFP) to label mitochondria (Clontech, Saint-Germain-en-Laye, France), according to manufacturer's guidelines (Neon transfection system; Life Technologies, Thermo Fisher Scientific, Basingstoke, UK). The second model included VL-17A cells transiently transfected with a plasmid expressing RFP to label mitochondria and a Drp1 mutant (Lys 38 to Ala) that acts as dominant negative (Drp1-K38A) and disables the fragmentation activity of Drp1 ${ }^{9,10}$ (pcDNA3-Drp1-K38A was a kind gift of Prof. Luca Scorrano, University of Padova, Padova, Italy). The third model included VL-17A cells stably expressing Drp1-K38A and/or enhanced green fluorescent protein (eGFP) generated through lentiviral transduction.

\section{Animal Model}

Drp1 (Dnm1l) Flox/Flox mice (C57BL/6/129) were generated, as described previously, ${ }^{16}$ and crossed with albumin-Cre mice (Alb-Cre; C57BL/6; Jackson Laboratory, Bar Harbor, ME) to obtain mice with liver-specific inactivation of Drpl (Dnmll) (Alb-Cre ${ }^{+}$Drp1) and compared with wild-type (WT; Alb-Cre ${ }^{-}$Drp1) male C57BL/6 J mice (Jackson Laboratory). Alb-Cre ${ }^{-}$Drp1 and Alb-Cre ${ }^{+}$Drp1 mice were divided into two groups and fed with either control diet or the ethanol diet National Institute on Alcohol Abuse and Alcoholism model [10-day ad libitum oral feeding with the Lieber-DeCarli ethanol liquid diet $(5 \% \mathrm{v} / \mathrm{v})$ plus a single binge ethanol feeding via gavage $(5 \mathrm{~g} / \mathrm{kg})]$, as previously described. ${ }^{17}$ All animals received humane care. All procedures were approved by the Institutional Animal Care and Use Committee of the University of Kansas Medical Center (Kansas City, KS). Alanine aminotransferase levels were measured in the blood of mice using a commercial kit (Liquid ALT Reagents; Pointe Scientific, Canton, MI).

\section{Plasmids, Lentiviral Preparation, and Infection}

The bicistronic lentiviral vector pULTRA expressing eGFP was a gift from Malcolm Moore (Memorial Sloan-Kettering Center, New York, NY; Addgene plasmid number 24129), ${ }^{18}$ and pULTRA-eGFP-Drp1-K38A was obtained from cloning the Drp1-K38A DNA fragment isolated from pcDNA3Drp1-K38A between BamHI and EcoRI restriction sites of pULTRA-eGFP and confirmed by nucleotide sequencing. Lentiviral preparations were obtained, as previously described. ${ }^{19}$ Supernatants were harvested and concentrated, and lentiviral titer was measured with QuickTiter Lentivirus Titer Kit (Cambridge Bioscience, Cambridge, UK), then used to infect the VL-17A cell line in the presence of $8 \mu \mathrm{g} / \mathrm{mL}$ polybrene (Sigma, Gillingham, UK).

\section{Confocal Microscopy}

Mitochondria were visualized in VL-17A cells using immunostaining, as previously described, ${ }^{7}$ after staining with antiTom20 (BD Biosciences, Oxford, UK) and NorthernLights anti-mouse IgG-NL557 (R\&D Systems, Minneapolis, MN) or in VL-17A cells expressing RFP to label mitochondria with or without transient expression of Drp1-K38A. Confocal images were collected using the PerkinElmer spinning-disk confocal microscope UltraVIEW (Perkin Elmer, Coventry, UK). 


\section{Morphologic Analysis}

Approximately 50 images (300 VL-17A cells) per condition were collected by confocal microscopy, and cells were ranked according to their mitochondrial phenotype and blindly scored (E.P.) using previously reported guidelines. ${ }^{7,10}$ The three cellular phenotypes (Figure 1A) included i) hyperfragmented: cells presenting $>50 \%$ of mitochondria as small and spherical entities (diameter, $<1 \mu \mathrm{m}$ ); ii) mixed network: cellular presentation of interconnected mitochondrial network with $<50 \%$ fragmented organelles and elongated mitochondria (length, $>2 \mu \mathrm{m} /$ width, $<1 \mu \mathrm{m}$ ) concurrently; and iii) hyperfused (megamitochondria): overinterconnected mitochondrial structure with abnormal enlargements, including discrete oversized megamitochondria with width and length $>1 \mu \mathrm{m}$, according to previously reported categorization recommendations. ${ }^{20}$

\section{Cell Growth and Viability Assays}

Cell growth was assessed at every passage by evaluating the number of viable cells [propidium iodide (-)] with growth rate calculated as fold increase per day. Cell proliferation was evaluated using the CellTrace Far Red Cell Proliferation Kit (Thermo Fisher Scientific), according to the manufacturer's guidelines. Early apoptotic or necrotic events were detected by flow cytometric analysis, with V450 Annexin V (BD Biosciences; mark of apoptosis) in addition to propidium iodide (Sigma) or 7-aminoactinomycin D (Biolegend, London, UK) staining. Data were acquired and analyzed on a BD FACSCanto II flow cytometer using BD FACSDiva software version 6.1 (BD Biosciences).

\section{Mitochondrial Isolation}

VL-17A cells were homogenized in isolation buffer $(250$ $\mathrm{mmol} / \mathrm{L}$ sucrose, $10 \mathrm{mmol} / \mathrm{L}$ tris, and $1 \mathrm{mmol} / \mathrm{L}$ EGTA, $\mathrm{pH}$ 7.4) using the Precellys 24 grinder (Bertin Technologies, Montigny le Bretonneux, France) with ceramic bead vials. Mitochondria were then isolated by standard differential centrifugations, as previously described. ${ }^{21}$ Mitochondrial protein concentration was quantified by Pierce BCA Protein Assay Kit (Thermo Fisher Scientific).

\section{Western Blot Analysis}

Protein extracts were obtained from total cell lysate, mitochondrial fraction, and mouse liver homogenate and separated by $12 \%$ SDS-PAGE, transferred onto nitrocellulose membranes (GE Healthcare Bio-Sciences AB, Uppsala, Sweden),
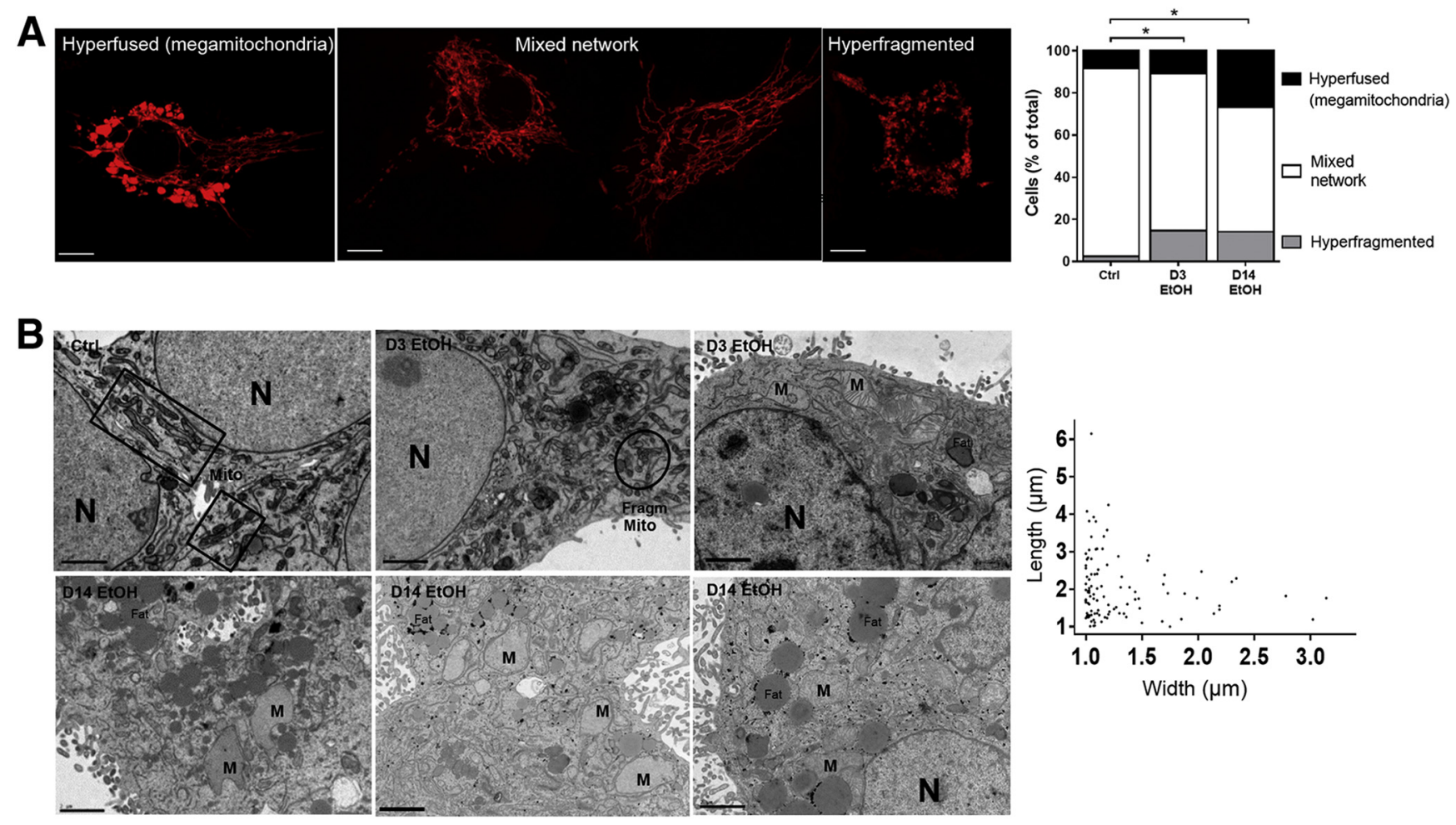

Figure 1 Ethanol (EtOH) severely affects mitochondrial (Mito) morphology in VL-17A cells. A: After mitochondrial immunostaining using anti-Tom20 antibody, cells were classified according to their mitochondrial phenotype and examples of the three groups are shown. The distribution among the three categories was compared between untreated cells (Ctrl) and cells exposed to 3 or 14 days of EtOH treatment (D3 or D14, respectively). A total of 300 cells were counted. B: Intracellular structure [nuclei $(\mathrm{N})$ ] and megamitochondria (M) development were analyzed in Ctrl or in day $3 /$ day 14 Et0H cultures. Representative electron micrographs show a mix of fragmented (Fragm; indicated by circles) and elongated (indicated by squares) organelles in the Ctrl and fragmented or megamitochondria (width, length $>1 \mu \mathrm{m}$ ) after EtOH exposure, especially at D14. The graph shows the distribution of the size of megamitochondria in approximately 100 cells. ${ }^{*} P<0.05$. Scale bars: $10 \mu \mathrm{m}(\mathbf{A}) ; 2 \mu \mathrm{m}(\mathbf{B})$. 

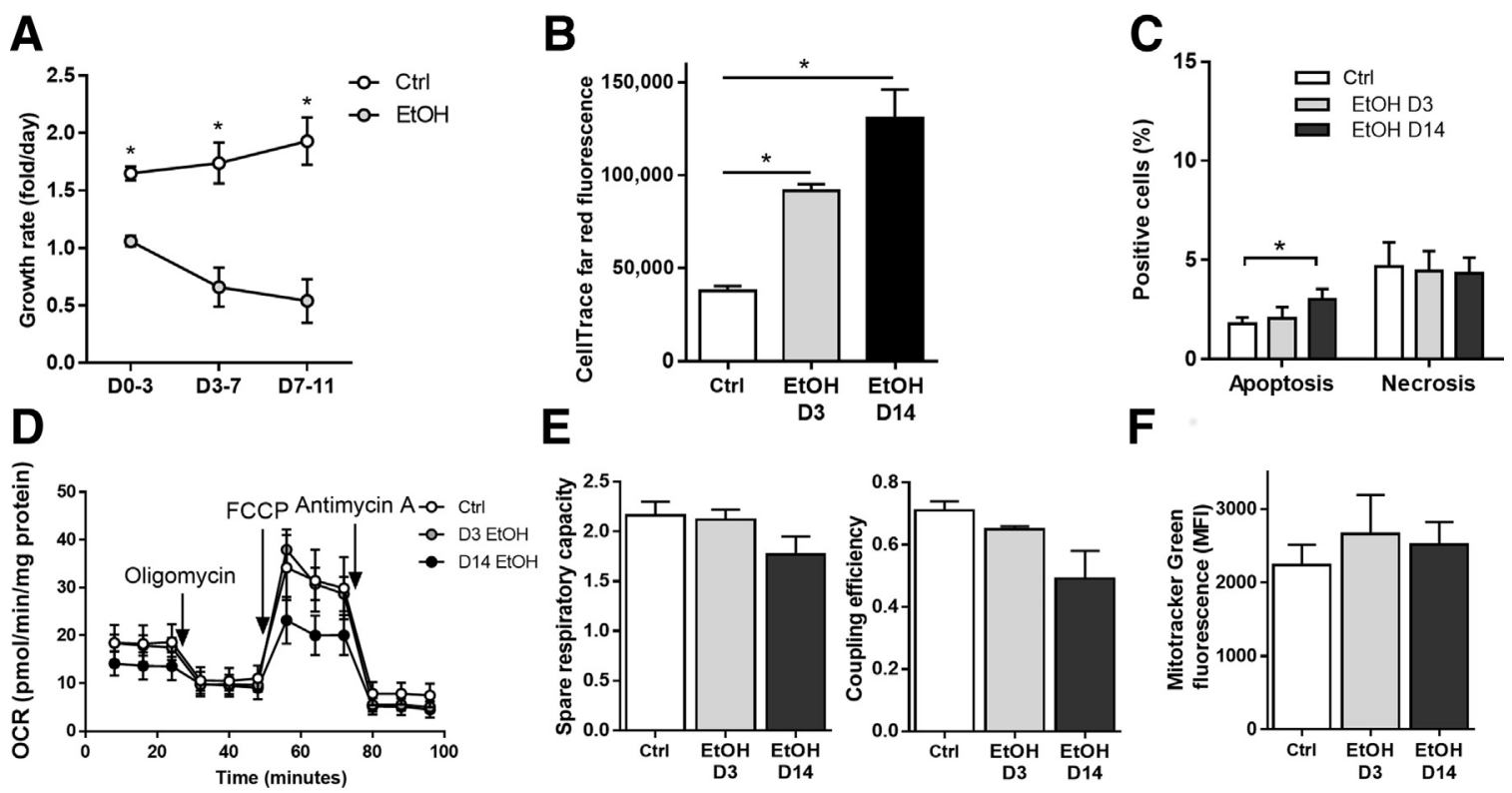

Figure 2 Ethanol (EtOH) exposure moderately reduces cell viability and severely affects cell growth, with no impact on mitochondrial respiration and mitochondrial mass in VL-17A hepatoma cell line. A: Cell growth rate decreases during culture in the presence of Et0H compared with the untreated control (Ctrl). B: EtOH induces growth arrest, as shown by changes in CellTrace dye fluorescence. C: Apoptotic [annexin- $\mathrm{V}(+)$ ] and necrotic [propidium iodide $(+)$ ] cells (positive cells) were quantified after 3 days (D3; $P>0.05$ ) or 14 days (D14) of EtOH exposure and compared with the untreated controls (Ctrl). The longer treatment induces a small but significant increase in apoptosis. D: The mitochondrial functionality was investigated, quantifying the oxygen consumption rate (OCR) in response to oligomycin, carbonyl cyanide 4-(trifluoromethoxy) phenylhydrazone (FCCP), or antimycin A; and no significant differences are reported between the control and the EtOH-treated cells $(P>0.05)$. E: The coupling efficiency and spare respiratory capacity were calculated, and no significant difference is observed between EtOH and Ctrl. F: The mitochondrial mass was analyzed after D3/D14 EtOH exposure, with no differences detected. Data are expressed as means \pm SEM. $n=4(\mathbf{A}$ and $\mathbf{B}) ; n=12$ (C, D3); $n=7$ (C, D14); $n=3(\mathbf{D}-\mathbf{F}) .{ }^{*} P<0.05$. D, days of treatment; MFI, mean fluorescence intensity.

and analyzed as previously described. ${ }^{7}$ The following antibodies (dilution 1:1000) were used: anti-Drp1, anti-Tom20, anti-MiD51 (SMCR7L/MID51; ProteinTech Europe, Manchester, UK), anti- mitochondrial fission factor (Sigma), anti-mitofusin-1, anti-mitofusin-2, anti-pyruvate dehydrogenase kinase 1, anti-actinin (Santa Cruz Biotechnology, Dallas, TX), anti- $\beta$-actin, and anti-voltage-dependent anion channel 1/porin (Abcam, Cambridge, UK).

\section{Mitochondrial Mass}

The mitochondrial mass was quantified by flow cytometry after staining the living cells with $20 \mathrm{nmol} / \mathrm{L}$ MitoTracker Green FM (Life Technologies, Thermo Fisher Scientific) for 30 minutes at $37^{\circ} \mathrm{C}$, according to the manufacturer's protocol, in presence of $2 \mu \mathrm{g} / \mathrm{mL}$ cyclosporin H (Santa Cruz Biotechnology). Data were acquired and analyzed on a BD FACSCanto II flow cytometer using BD FACSDiva software version 6.1.

\section{Mitochondrial Respiration}

Seahorse XF24 Analyzer (Seahorse Bioscience, Copenhagen, Denmark) was used to measure the mitochondrial oxygen consumption rate (OCR) in untreated/treated VL-17A cells in situ. Changes in OCR (nmoles/minute) in response to oligomycin (1 $\mu \mathrm{g} / \mathrm{mL}$; Sigma), carbonyl cyanide 4-(trifluoromethoxy) phenylhydrazone (FCCP; $0.6 \mu \mathrm{mol} / \mathrm{L}$; Sigma), and antimycin A ( $2 \mu \mathrm{mol} / \mathrm{L}$; Sigma) were measured and used to calculate the spare respiratory capacity $\left(\mathrm{OCR}_{\mathrm{FCCP}} /\right.$ $\left.\mathrm{OCR}_{\text {basal }}\right)$ and the coupling efficiency (1-OCR oligomycin ' $\left.\mathrm{OCR}_{\text {basal }}\right)$. Measurements were repeated in triplicate in three separate experiments, and the OCR values were normalized for the total protein content, quantified by Pierce BCA Protein Assay Kit (Thermo Fisher Scientific).

\section{Electron Microscopy}

Cells or murine liver tissues were fixed with $2 \%$ paraformaldehyde and $1.5 \%$ glutaraldehyde in $0.1 \mathrm{~mol} / \mathrm{L}$ cacodylate buffer or $2.5 \%$ glutaraldehyde in $0.1 \mathrm{~mol} / \mathrm{L}$ phosphate buffer (pH 7.4), followed by $1 \% \mathrm{OSO}_{4}$. After dehydration, ultrathin sections were stained with uranyl acetate and lead citrate and viewed in a JEM 1011CX or 1010 transition electron microscope (JEOL USA, Peabody, MA), as described previously. ${ }^{22}$ The size of megamitochondria was evaluated in the cells using ImageJ software version 1.49c (NIH, Bethesda, MD; http://imagej.nih.gov/ij): images were analyzed in blind (E.P.), and the length and the width of megamitochondria $(>1 \mu \mathrm{m})$ were measured using the straight line function across each organelle, followed by the ImageJ function "analyze measure" to obtain the numeric values of length and width.

\section{Statistical Analysis}

Continuous variables were expressed as means \pm SEM, and categorical variables were described as means of counts and 

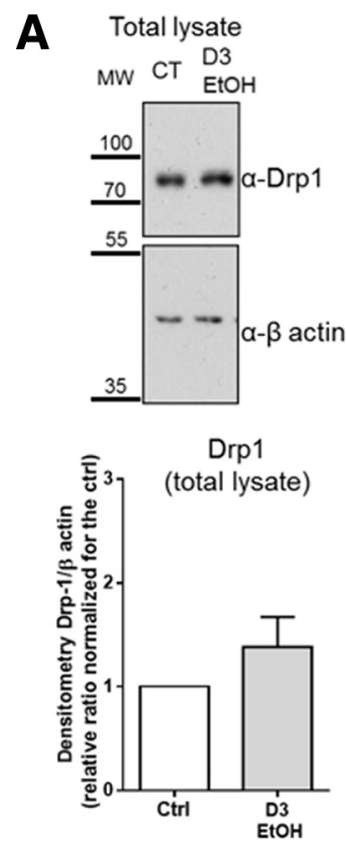

Isolated mitochondria

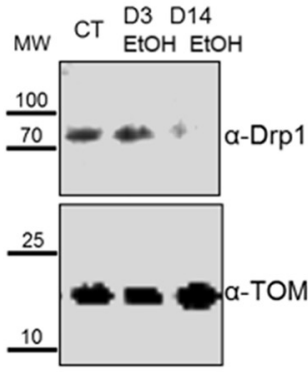

Drp1
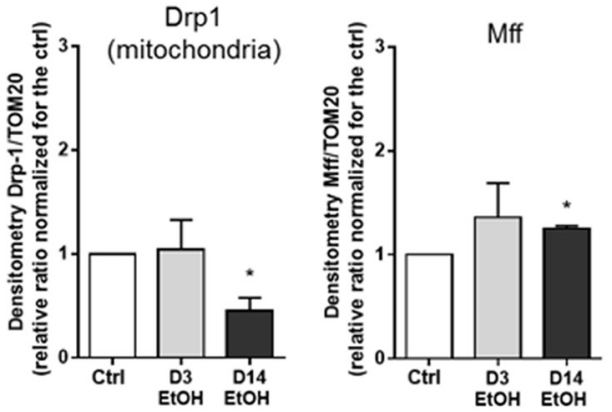
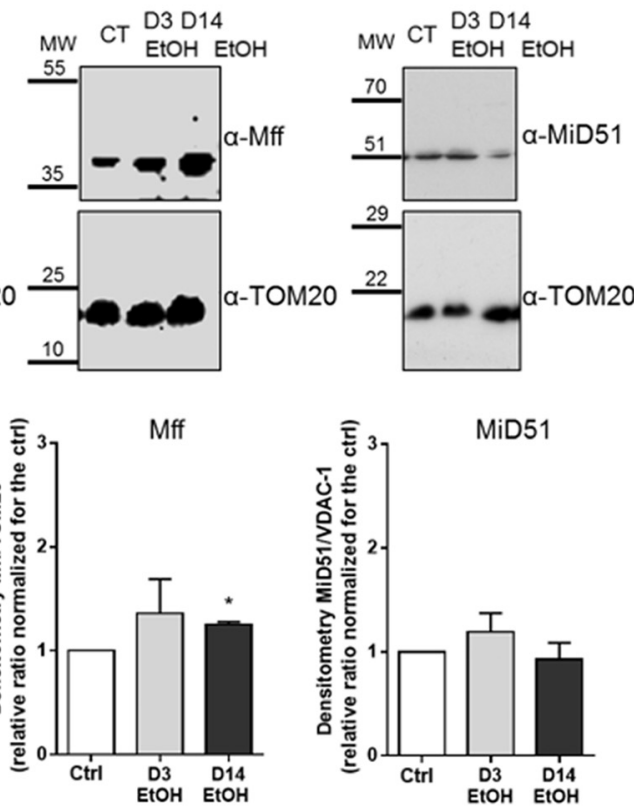

B
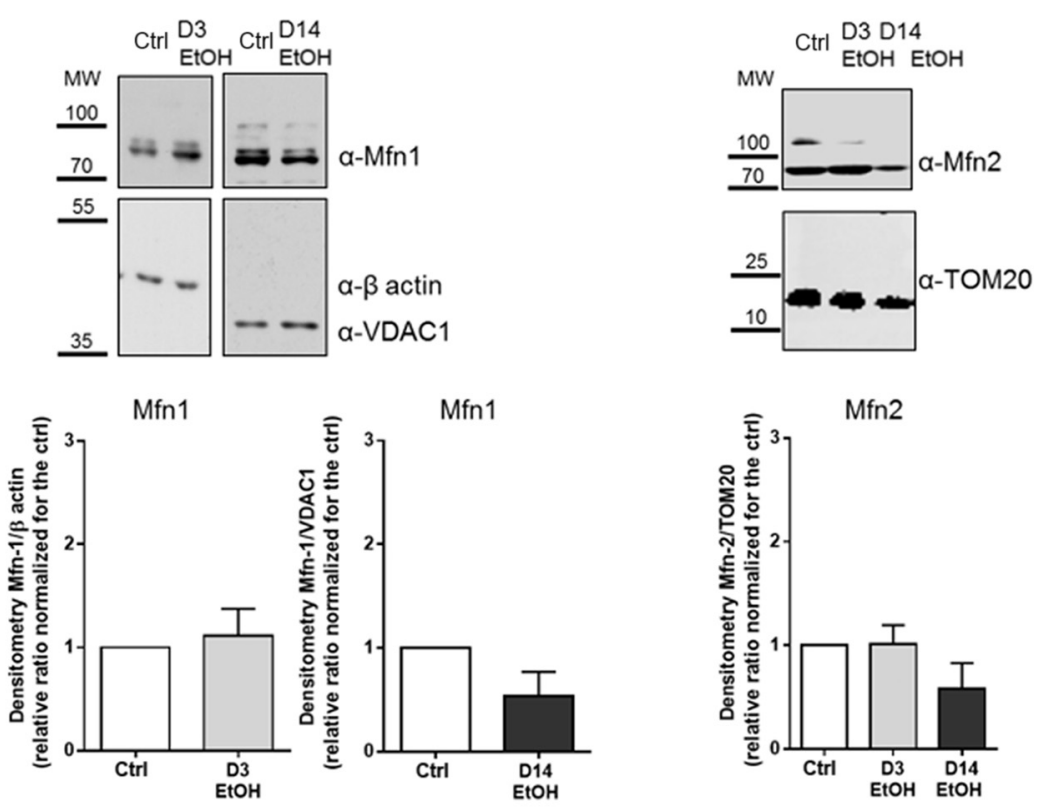

C
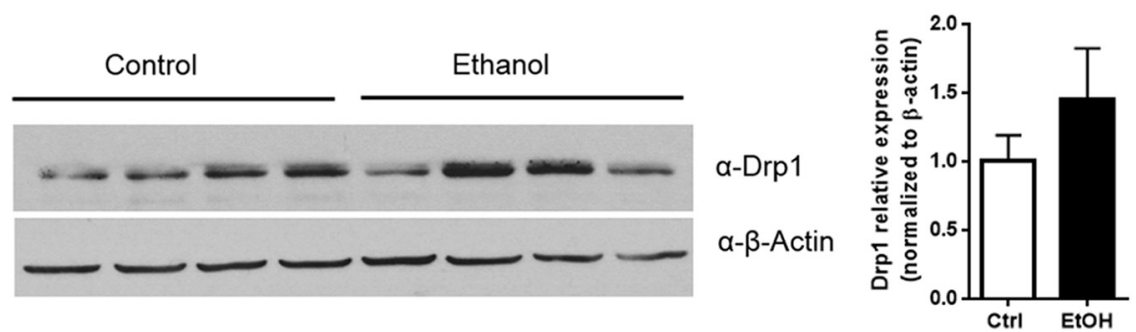

Figure 3 Ethanol (EtOH) exposure perturbs the Drp1 pathway in VL-17A cells. A: Mitochondrial fragmentation: The expression or localization of Drp1 and its receptors [mitochondrial fission factor (Mff) and MiD51] was analyzed in the ethanol cultures after 3 or 14 days of EtOH exposure (D3 or D14). B: Mitochondrial fusion: The expression of mitofusin-1 (Mfn1) and mitofusin-2 (Mfn2) was evaluated in untreated VL-17A cells and after D3 or D14. C: The hepatic expression of Drp1 was analyzed on wild-type mice fed with control (Ctrl) or ethanol diet. In all panels, representative Western blots are shown along with the densitometric analysis. Data are expressed as means \pm SEM. $n>3(\mathbf{A}$ and $\mathbf{B}) ; n=4(\mathbf{C}) .{ }^{*} P<0.05$ compared with Ctrl. MW, molecular weight. 
percentages. Comparisons were performed using independent or paired $t$-test or nonparametric Friedman's analysis of variance for repeated measures, when multiple treatment groups were analyzed simultaneously. Categorical variables (morphologic analysis) were analyzed by $\chi^{2}$ test. Statistics were calculated using MS Excel 2010 (Microsoft, Redmond, WA) and GraphPad Prism software version 6.07 (GraphPad, La Jolla, CA). Statistical significance was set at $P<0.05$.

\section{Results}

Ethanol Exposure Severely Impairs Cellular

Proliferation and Mitochondrial Morphology in VL-17A

Cells, with No Impact on Mitochondrial Mass or

Functionality

A significant impact on proliferative potential was observed in response to both short and long exposure of ethanol in VL-17A cells (Figure 2, A and B). After the short insult, the apoptotic and necrotic levels were not significantly different from the controls (Figure 2C), whereas longer alcohol exposure induced a slight, but significant, increase in the percentage of apoptotic cells (Figure 2C). The measurement of basal mitochondrial respiration rate (Figure 2D) and the calculation of the coupling efficiency as well as of the spare respiratory capacity were not different between the short- and long-ethanol cultures compared with the controls (Figure 2E). The mitochondrial mass was also not affected by ethanol exposure (Figure 2F), but remarkable mitochondrial remodeling was observed by confocal and electron microscopy. The untreated cells presented a prevalence of interconnected mitochondrial network, with a balance between fusion/fission, whereas the ethanol-treated cells displayed a significantly different distribution among the three mitochondrial phenotypes both in day 3 and day 14 cultures (Figure 1A), with $>14 \%$ of the cells exposed to ethanol showing a hyperfragmented network. With extended exposure to ethanol, the presence of oversized and misshapen megamitochondria (Figure 1B) was observed. These abnormalities were also detected by confocal microscopy (Figure 1A), in which almost 30\% of the cells exposed to 14 days of ethanol were classified in the hyperfused/megamitochondria phenotype.

\section{Extended Ethanol Exposure Affects Mitochondria- Shaping Protein Expression in VL-17A Cells}

Short ethanol exposure in VL-17A cells did not impact the fusion or fragmentation pathways (Figure 3), but prolonged ethanol treatment significantly reduced the mitochondrial translocation of Drp1, the main protagonist driving mitochondrial fragmentation; this was paralleled with an observed increase in the Drp1 receptor mitochondrial fission factor (Figure 3A). Ethanol did not affect the fusion machinery; no changes were observed in mitofusin-1 or mitofusin-2 (Figure 3B). In WT mice fed with ethanol, no significant changes in Drp1 hepatic expression were detected when compared with control animals (Figure 3C).

\section{Drp1 Inactivation Promotes Megamitochondria Formation and Abrogates Ethanol-Induced Cell Growth Retardation in Hepatoma Cells}

The analysis of the mitochondrial phenotypes (hyperfragmented, mixed, and hyperfused) and the evaluation of ethanol toxicity were performed in the cellular model in which the fragmentation pathway was disabled (Drp1-K38A) in comparison to the control VL-17A with active Drp1. In the absence of ethanol, inactivation of Drp1 led to a notable increase in the appearance of hyperfused megamitochondria (Figure 4A). Ethanol induced fragmentation in the control VL17A cells (Figure 4B), but this was completely abolished in the Drp1-K38A cells, which presented an additional increase in megamitochondria formation (Figure 4A). Further to this, the impact of ethanol on cellular growth was evaluated in the stable lines of VL-17A expressing both eGFP and Drp1-K38A $\left(\mathrm{GFP}^{+}\right.$Drp1-inactive) and the control cells expressing only eGFP (WT Drp1-active). In cultures with Drp1, active growth retardation was observed during ethanol exposure (Figure 4C). However, inactivation of Drp1, with megamitochondria formation, rescued this growth blockade, with percentage of $\mathrm{GFP}^{+} / \mathrm{Drp} 1-\mathrm{K} 38 \mathrm{~A}$ cells significantly increasing over time despite alcohol insult. Moreover, these cells showed a proliferative advantage compared with the $\mathrm{GFP}^{-}$cells in the same culture (Figure 4C).

\section{Drp1 Inactivation Causes Megamitochondria Development in Murine Livers}

The hepatic mitochondrial evaluation by electron microscopy in the mice after liver inactivation of Drp1 showed the formation of enlarged and round organelles (Figure 5, A and B), with the same morphologic characteristics of megamitochondria observed in the cellular model after ethanol exposure (Figure 1) as well as previously reported in animal models fed with alcohol ${ }^{23}$ or liver biopsies from alcoholic patients. ${ }^{24}$ The incidence of megamitochondria was significantly increased in the liver of these mice after inactivation of Drp1 (Alb-Cre ${ }^{+}$Drp1) compared with the WT (Alb$\mathrm{Cre}^{-}$Drp1) (Figure 5C).

Ethanol Exposure Causes Megamitochondria Formation in Murine Livers, and Drp1 Inactivation Exacerbates This Phenomenon and Reduces Ethanol-Induced Hepatotoxicity

In both WT mice (Alb-Cre ${ }^{-}$Drp1) and mice with inactive hepatic Drp1 (Alb-Cre ${ }^{+}$Drp1), the ethanol diet induced a significant increase in the percentage of megamitochondria (Figure 5, A-C), and this effect was significantly more pronounced in mice with inactive Drp1 when compared with WT (Figure 5C). The effect of ethanol on alanine 

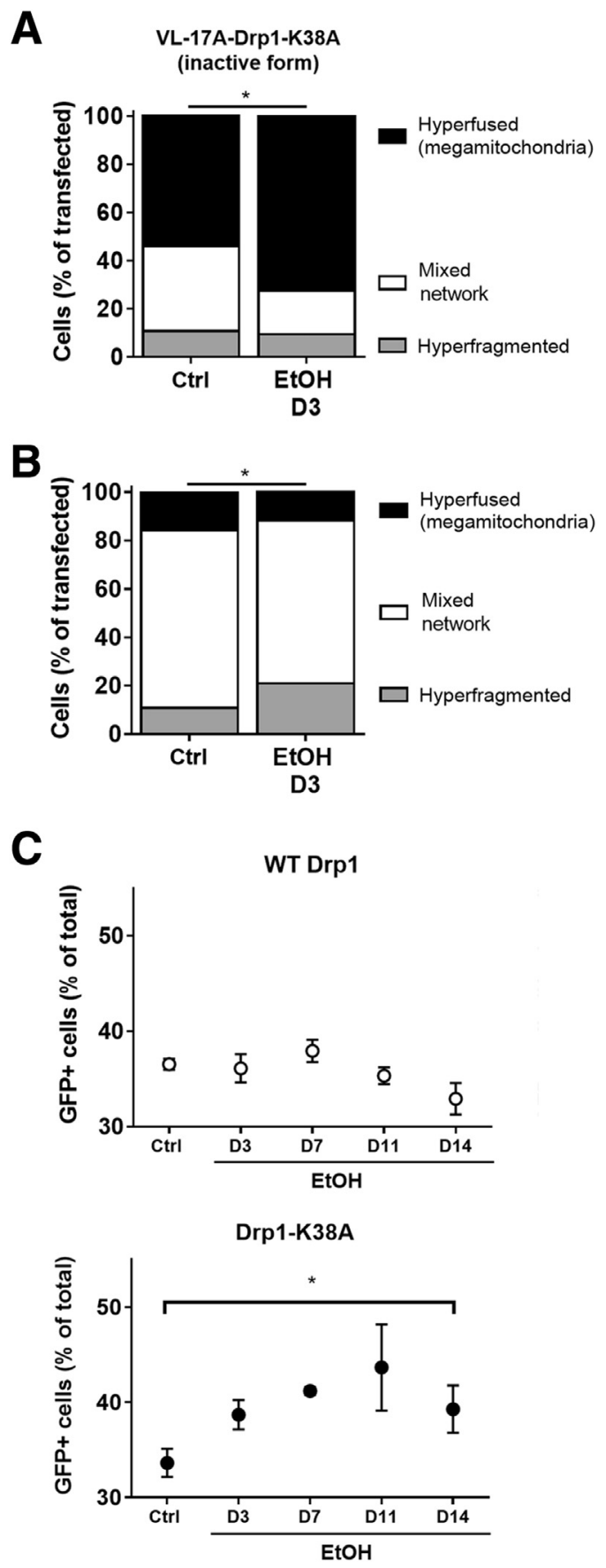

Figure 4 Drp1 inactivation promotes megamitochondria formation and reduces ethanol-induced toxicity in hepatoma cells. A: Approximately 120 cells [positive for mitochondrial matrix red fluorescent protein (mtRFP) and expressing Drp1-K38A (inactive form)] with or without 3 days of ethanol (EtOH) exposure were sorted into three categories according to their mitochondrial morphology (hyperfused/megamitochondria, mixed network, or hyperfragmented), and a big proportion of cells with megamitochondria was detected. B: The three mitochondrial phenotypes were analyzed in VL17A cells expressing wild-type (WT) Drp1 and positive for mtRFP (approximately 300 cells counted per condition). The distribution was different after 3 days of EtOH exposure (D3) compared with untreated control (Ctrl). C: In VL-17A stably expressing enhanced green fluorescent protein [eGFP (WT Drp1)] or coexpressing eGFP and Drp1-K38A (Drp1-K38A), the number of $\mathrm{GFP}^{+}$cells was quantified during 14 days of $\mathrm{EtOH}$ exposure. A significant increase was detected only in Drp1-K38A, but not in WT Drp1. Data are expressed as means \pm SEM. $n=3$ (C). ${ }^{*} P<0.05$. D, day. aminotransferase levels was also analyzed, and these values were found to be significantly increased in WT mice, but not in mice with inactive Drp1, as shown from the ratios of ethanol versus control diet (Figure 5D).

\section{Discussion}

One of the main findings of this study was the profound impact of ethanol on hepatic mitochondrial morphology, including both organelle fragmentation and enlargement. Both short and long exposure of ethanol induced an increase in the hyperfragmented mitochondrial fraction, confirming ethanol-related augmented fission, which has previously only been reported in other cell types, including human retinal pigment epithelial cells ${ }^{25,26}$ and alveolar macrophages. ${ }^{27}$ In the hepatoma cell model, ethanol exposure caused an immediate change in mitochondrial shape, with significant imbalance of the fusion/fission equilibrium, which was confirmed by the changes in mitochondria-shaping protein expression observed. These results suggest that mitochondrial dynamics play an essential role in alcohol-related liver disease and support other studies that have observed hepatic mitochondrial remodeling during alcoholic insults. ${ }^{28,29}$ In addition, after the prolonged exposure, hyperfragmentation was accompanied by the formation of disproportionately enlarged megamitochondria. These architectural alterations in hepatic mitochondria were similar to the presentation of enlarged and hyperfused organelles previously identified in patients and animal models, as one of the consequences of chronic ethanol consumption. Indeed, the detection of megamitochondria in liver biopsies has been regarded as a specific hallmark for alcohol-related liver disease since the 1970 s. $^{23,30,31}$ In this study, extended ethanol exposure induced the formation of megamitochondria in the VL-17A cells, endorsing, for the first time, the validity of this cell line in studying the phenomenon.

These data suggest that the appearance of megamitochondria after a prolonged/chronic stimulus alongside mitochondrial hyperfragmentation is an adaptive mechanism possibly to counteract excessive fission and mitophagy, which unregulated would be highly detrimental to cellular survival. $^{32,33}$ This seems to represent a response of the hepatocytes to preserve cellular function in the face of a toxic insult, with mitochondrial fragmentation facilitating the clearance of dysfunctional organelles via mitophagy. This is supported by reports demonstrating that alcohol stimulates the initiation of autophagy ${ }^{34,35}$ and that damaged mitochondria and accumulated lipid droplets are the selective target of autophagy induced by acute ethanol exposure. ${ }^{36}$

In addition, this study identified, for the first time, the master fission mediator Drp1 as the key player involved in the alcohol-related morphologic changes. In our cellular model, the expression of mutated dominant-negative Drp1 (Drp1-K38A) was sufficient to abolish the ethanol-induced mitochondrial hyperfragmentation, and one of the most 
A

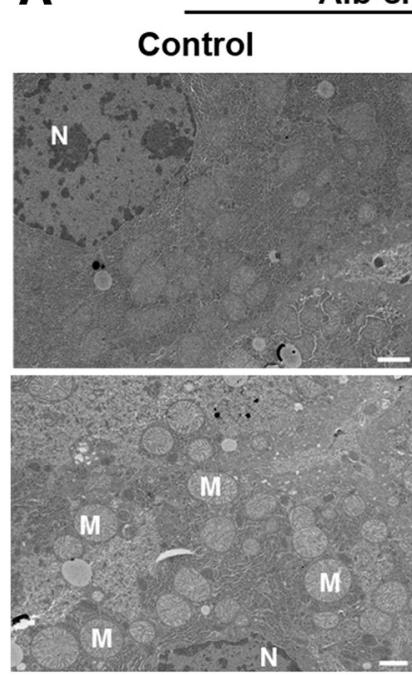

Alb-cre-Drp1
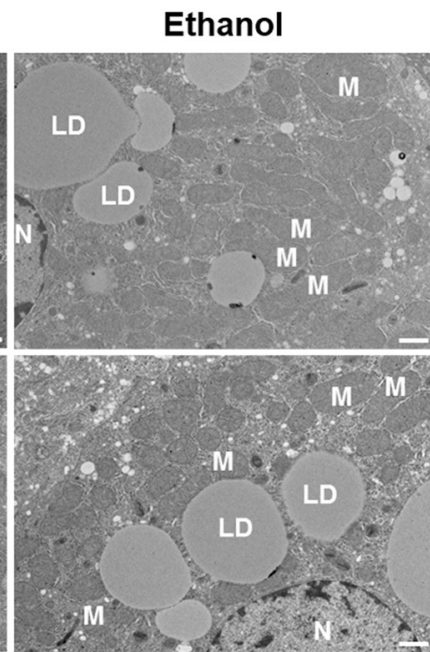

C

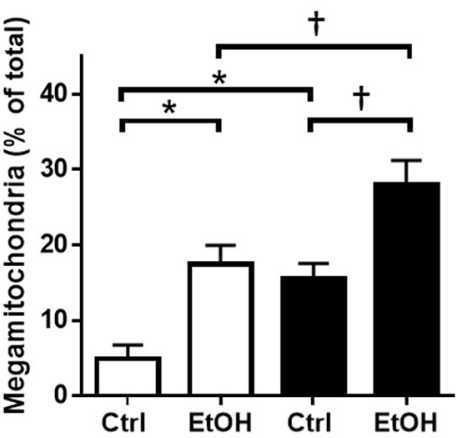

B Alb-cre+Drp1

Ethanol

M

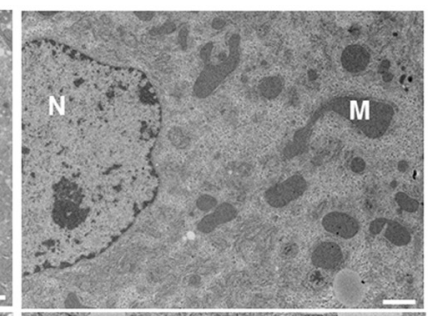

Control

Ethanol
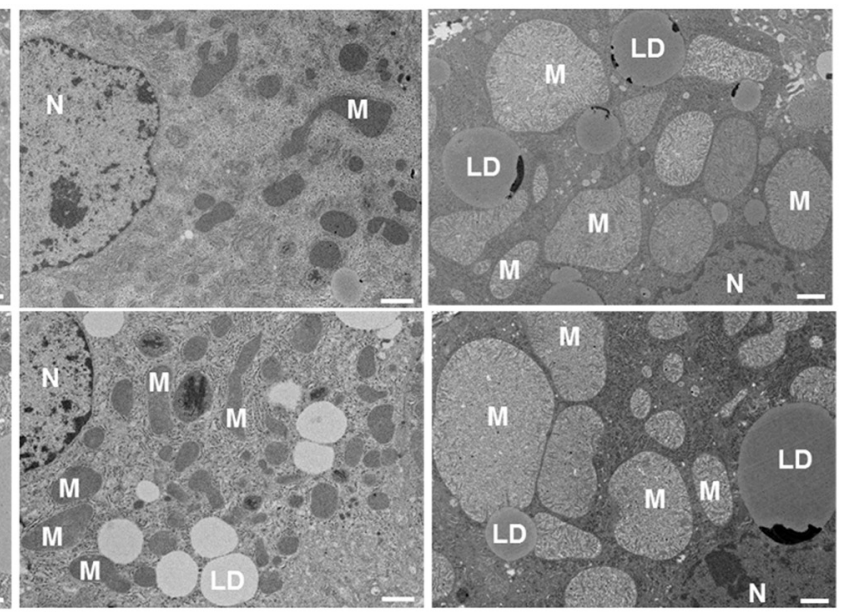

D

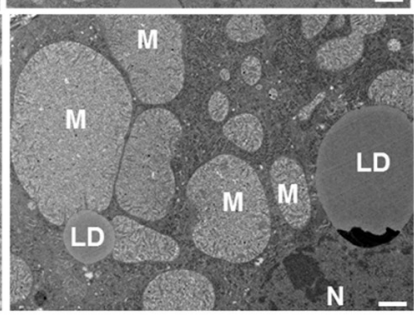

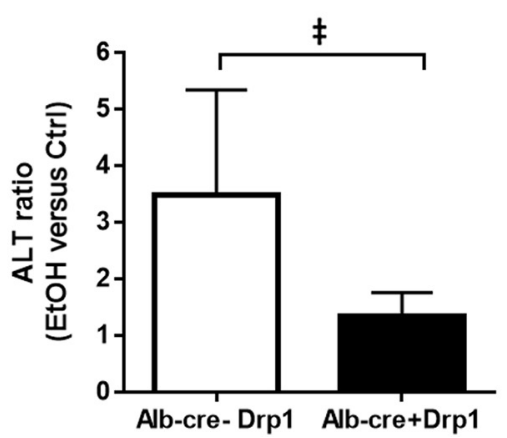

Figure 5 Ethanol (EtOH) exposure causes megamitochondria development in mice, and hepatic Drp1 inactivation increases this effect and reduces ethanolinduced hepatotoxicity. Megamitochondria formation was analyzed in mice with liver inactivation of Drp1 (Alb-Cre ${ }^{+}$Drp1) and compared with wild-type mice (Alb$\mathrm{Cre}^{-} \mathrm{Drp1}$ ) exposed to control (Ctrl) or ethanol diet. A and B: Representative electron micrographs show examples of megamitochondria (M; width, length $>2 \mu \mathrm{m}$ ) in Alb-Cre ${ }^{-} \operatorname{Drp1}$ (A) and Alb-Cre ${ }^{+} \operatorname{Drp1}$ (B) fed with control or ethanol diet. C: The percentage of megamitochondria in Alb-Cre ${ }^{-}$Drp1 and Alb-Cre ${ }^{+}$Drp1 was quantified: a significant increase after ethanol exposure is seen in both groups, and this effect significantly exacerbates after Drp1 inactivation ( $>360$ mitochondria analyzed). D: Alanine aminotransferase (ALT) levels in the blood of Alb-Cre ${ }^{-}$Drp1 and Alb-Cre ${ }^{+}$Drp1 mice after exposure to ethanol were quantified, and the ratios versus ALT in mice fed with control diet shown (ratio of mean \pm error propagation). Data are expressed as means $\pm \mathrm{SEM} . n>3$ (C and $\mathbf{D})$ ) ${ }^{*} P<0.05$ versus Alb-Cre ${ }^{-}$Drp1 Ctrl diet; ${ }^{\dagger} P<0.05$ versus Alb-Cre ${ }^{+}$Drp1 ethanol diet; ${ }^{\ddagger} P<0.05$. Scale bar $=1 \mu \mathrm{m}$ (A and B). LD, lipid droplet; $\mathrm{N}$, nucleus.

striking results was the beneficial effect on cell proliferation of Drp1 inhibition in response to ethanol insult. In control cells with active Drp1, ethanol was able to induce a significant growth retardation even after 3 days of exposure. Indeed, ALD is known to be associated with a significant loss of hepatocyte proliferation and impaired regeneration, further driving the inability of the liver to deal with the toxic insult because of a reduced capacity to replace dying hepatocytes. This is an area of much interest, and many clinical trials are trying to find ways to promote hepatocyte proliferation. ${ }^{37}$ Drp1 inactivation in the VL-17A cells showed a protective effect with the rescue of cell growth retardation induced by ethanol both at 3 and 14 days of toxic exposure. The toxic effect in terms of cell death was probably too mild to be appreciated in this model (data not shown). More important, Drp1 inactivation showed a beneficial effect also in the mouse model, in which the ethanol exposure was associated with lower alanine aminotransferase levels. This finding is particularly relevant considering the significant and escalating clinical burden of alcohol-related liver disease and therefore the urgent need to develop specific and targeted drugs. Currently, the cornerstone of therapy remains abstinence with supportive treatments and few other options with limited efficacy. ${ }^{38}$ There are no therapies aimed at stopping ongoing ethanol toxicity in hepatocytes, to halt injury and allow time for regeneration and repair. This study gives the initial experimental evidence that targeting mitochondrial dynamics could reduce liver injury by improving survival adaptations of hepatocytes to alcohol toxicity. Moreover, it has been recently demonstrated that Drp1 inhibition has a beneficial and protective effect in several other models of liver injury, including hepatotoxicity induced by toxic agents (senecionine $^{39}$ and cadmium $^{40}$ ), in animal models of dietinduced steatosis ${ }^{41}$ and cholestatic liver injury. ${ }^{42}$

The most important finding of this study is that Drp1 inhibition is able to cause a rapid and extensive formation of megamitochondria, which was confirmed in mouse models 
with liver-specific inactivation of Drp1. The molecular mechanisms regulating the development of these morphologic abnormalities and their influence on cellular responses have not been elucidated to date, but several clinical studies have endorsed the presence of intrahepatic megamitochondria in ALD as beneficial clinical indicators. ${ }^{43-46}$ Moreover, a recent study has reported that the treatment with Drp1 inhibitor Mdivi-1 in Triticum aestivum caused fission retardation with consequent induction of megamitochondria, and this has been recognized as a strategic adaptation of plants to stress. ${ }^{47}$ The prospect for the use of Drp1 inhibitors in vivo has also become more promising after the demonstration of their prophylactic and therapeutic effects in several models of tissue injury, induced by toxic insult or ischemia/reperfusion damage. ${ }^{48-53}$ The beneficial advantage of these agents in alcohol-induced liver injury may also be two pronged because of a decrease of oxidative stress, as associated with Drp1 inhibitor treatment in a murine cardiac arrest model,${ }^{54}$ which plays a fundamental role in alcohol-related hepatotoxicity.

\section{Acknowledgments}

We thank Mark Turmaine (University of London, London, UK) and Dahn Clemens (University of Nebraska/Veterans Affairs Medical Center, Lincoln, NE) for the technical support; Prof. Luca Scorrano (University of Padova, Padova, Italy) for providing pcDNA3-Drp1-K38A; and Malcolm Moore (Memorial Sloan-Kettering Center, New York, NY) for providing pULTRA-expressing enhanced green fluorescent protein.

E.P. designed the study, collected and analyzed the data, and wrote the manuscript; X.M., A.R., A.D., and S.W. performed the experiments and collected the data; V.I. performed experiments and analyzed the data; H.-.M.N. and H.S. generated the mouse model; R.W. designed the study and wrote the manuscript; W.-.X.D. collected and analyzed the data; and S.C. designed the study, analyzed the data, and wrote the manuscript.

\section{Supplemental Data}

Supplemental material for this article can be found at https://doi.org/10.1016/j.ajpath.2018.11.008.

\section{References}

1. Kaplan W: Priority medicines for Europe and the world "a public health approach to innovation": update on 2004 background paper 6.14, 1-71: harmful use of alcohol: alcohol use disorders and alcoholic liver diseases. Geneva, Switzerland, World Health Organization, February 8, 2013. Available at http://apps.who.int/medicine docs/documents/s20245en/s20245en.pdf (accessed March 2018)

2. Garcia-Ruiz C, Kaplowitz N, Fernandez-Checa JC: Role of mitochondria in alcoholic liver disease. Curr Pathobiol Rep 2013, 1:159-168
3. Nassir F, Ibdah JA: Role of mitochondria in alcoholic liver disease World J Gastroenterol 2014, 20:2136-2142

4. Zhu H, Jia Z, Misra H, Li YR: Oxidative stress and redox signaling mechanisms of alcoholic liver disease: updated experimental and clinical evidence. J Dig Dis 2012, 13:133-142

5. Youle RJ, van der Bliek AM: Mitochondrial fission, fusion, and stress. Science 2012, 337:1062-1065

6. Lee YJ, Jeong SY, Karbowski M, Smith CL, Youle RJ: Roles of the mammalian mitochondrial fission and fusion mediators Fis1, Drp1, and Opal in apoptosis. Mol Biol Cell 2004, 15:5001-5011

7. de Brito OM, Scorrano L: Mitofusin 2 tethers endoplasmic reticulum to mitochondria. Nature 2008, 456:605-610

8. Cereghetti GM, Stangherlin A, Martins de BO, Chang CR, Blackstone C, Bernardi P, Scorrano L: Dephosphorylation by calcineurin regulates translocation of Drp1 to mitochondria. Proc Natl Acad Sci U S A 2008, 105:15803-15808

9. Smirnova E, Shurland DL, Ryazantsev SN, van der Bliek AM: A human dynamin-related protein controls the distribution of mitochondria. J Cell Biol 1998, 143:351-358

10. Smirnova E, Griparic L, Shurland DL, van der Bliek AM: Dynaminrelated protein Drp1 is required for mitochondrial division in mammalian cells. Mol Biol Cell 2001, 12:2245-2256

11. Palmer CS, Osellame LD, Stojanovski D, Ryan MT: The regulation of mitochondrial morphology: intricate mechanisms and dynamic machinery. Cell Signal 2011, 23:1534-1545

12. Loson OC, Song Z, Chen H, Chan DC: Fis1, Mff, MiD49, and MiD51 mediate Drp1 recruitment in mitochondrial fission. Mol Biol Cell 2013, 24:659-667

13. Archer SL: Mitochondrial dynamics: mitochondrial fission and fusion in human diseases. N Engl J Med 2013, 369:2236-2251

14. Burte F, Carelli V, Chinnery PF, Yu-Wai-Man P: Disturbed mitochondrial dynamics and neurodegenerative disorders. Nat Rev Neurol 2015, 11:11-24

15. Donohue TM, Osna NA, Clemens DL: Recombinant Hep G2 cells that express alcohol dehydrogenase and cytochrome P450 2E1 as a model of ethanol-elicited cytotoxicity. Int J Biochem Cell Biol 2006, 38:92-101

16. Kageyama Y, Hoshijima M, Seo K, Bedja D, Sysa-Shah P, Andrabi SA, Chen W, Hoke A, Dawson VL, Dawson TM, Gabrielson K, Kass DA, Iijima M, Sesaki H: Parkin-independent mitophagy requires Drp1 and maintains the integrity of mammalian heart and brain. EMBO J 2014, 33:2798-2813

17. Chao X, Wang S, Zhao K, Li Y, Williams JA, Li T, Chavan H, Krishnamurthy P, He XC, Li L, Ballabio A, Ni HM, Ding WX Impaired TFEB-mediated lysosome biogenesis and autophagy promote chronic ethanol-induced liver injury and steatosis in mice. Gastroenterology 2018, 155:865-879

18. Lou E, Fujisawa S, Morozov A, Barlas A, Romin Y, Dogan Y, Gholami S, Moreira AL, Manova-Todorova K, Moore MA: Tunneling nanotubes provide a unique conduit for intercellular transfer of cellular contents in human malignant pleural mesothelioma. PLoS One 2012, 7:e33093

19. Iansante V, Choy PM, Fung SW, Liu Y, Chai JG, Dyson J, Del Rio A, D'Santos C, Williams R, Chokshi S, Anders RA, Bubici C, Papa S: PARP14 promotes the Warburg effect in hepatocellular carcinoma by inhibiting JNK1-dependent PKM2 phosphorylation and activation. Nat Commun 2015, 6:7882

20. Cascarano J, Chambers PA, Schwartz E, Poorkaj P, Gondo RE: Organellar clusters formed by mitochondrial-rough endoplasmic reticulum associations: an ordered arrangement of mitochondria in hepatocytes. Hepatology 1995, 22:837-846

21. Frezza C, Cipolat S, Scorrano L: Organelle isolation: functional mitochondria from mouse liver, muscle and cultured fibroblasts. Nat Protoc 2007, 2:287-295

22. Ni HM, Bockus A, Boggess N, Jaeschke H, Ding WX: Activation of autophagy protects against acetaminophen-induced hepatotoxicity. Hepatology 2012, 55:222-232 
23. Koch OR, Roatta de Conti LL, Bolanos LP, Stoppani AO: Ultrastructural and biochemical aspects of liver mitochondria during recovery from ethanol-induced alterations: experimental evidence of mitochondrial division. Am J Pathol 1978, 90:325-344

24. Iseri OA, Gottlieb LS: Alcoholic hyalin and megamitochondria as separate and distinct entities in liver disease associated with alcoholism. Gastroenterology 1971, 60:1027-1035

25. Bonet-Ponce L, Saez-Atienzar S, da CC, Flores-Bellver M, Barcia JM, Sancho-Pelluz J, Romero FJ, Jordan J, Galindo MF: On the mechanism underlying ethanol-induced mitochondrial dynamic disruption and autophagy response. Biochim Biophys Acta 2015, 1852:1400-1409

26. Flores-Bellver M, Bonet-Ponce L, Barcia JM, Garcia-Verdugo JM, Martinez-Gil N, Saez-Atienzar S, Sancho-Pelluz J, Jordan J, Galindo MF, Romero FJ: Autophagy and mitochondrial alterations in human retinal pigment epithelial cells induced by ethanol: implications of 4-hydroxy-nonenal. Cell Death Dis 2014, 5:e1328

27. Liang Y, Harris FL, Brown LA: Alcohol induced mitochondrial oxidative stress and alveolar macrophage dysfunction. Biomed Res Int 2014, 2014:371593

28. Das S, Hajnoczky N, Antony AN, Csordas G, Gaspers LD, Clemens DL, Hoek JB, Hajnoczky G: Mitochondrial morphology and dynamics in hepatocytes from normal and ethanol-fed rats. Pflugers Arch 2012, 464:101-109

29. Han D, Ybanez MD, Johnson HS, McDonald JN, Mesropyan L, Sancheti H, Martin G, Martin A, Lim AM, Dara L, Cadenas E, Tsukamoto H, Kaplowitz N: Dynamic adaptation of liver mitochondria to chronic alcohol feeding in mice: biogenesis, remodeling, and functional alterations. J Biol Chem 2012, 287:42165-42179

30. Bruguera M, Bertran A, Bombi JA, Rodes J: Giant mitochondria in hepatocytes: a diagnostic hint for alcoholic liver disease. Gastroenterology 1977, 73:1383-1387

31. French SW: Intragastric ethanol infusion model for cellular and molecular studies of alcoholic liver disease. J Biomed Sci 2001, 8:20-27

32. Lee SB, Kang JW, Kim SJ, Ahn J, Kim J, Lee SM: Afzelin ameliorates D-galactosamine and lipopolysaccharide-induced fulminant hepatic failure by modulating mitochondrial quality control and dynamics. Br J Pharmacol 2017, 174:195-209

33. Su YC, Qi X: Inhibition of excessive mitochondrial fission reduced aberrant autophagy and neuronal damage caused by LRRK2 G2019S mutation. Hum Mol Genet 2013, 22:4545-4561

34. Thomes PG, Trambly CS, Thiele GM, Duryee MJ, Fox HS, Haorah J, Donohue TM Jr: Proteasome activity and autophagosome content in liver are reciprocally regulated by ethanol treatment. Biochem Biophys Res Commun 2012, 417:262-267

35. Williams JA, Ni HM, Ding Y, Ding WX: Parkin regulates mitophagy and mitochondrial function to protect against alcohol-induced liver injury and steatosis in mice. Am J Physiol Gastrointest Liver Physiol 2015, 309:G324-G340

36. Ding WX, Li M, Chen X, Ni HM, Lin CW, Gao W, Lu B, Stolz DB, Clemens DL, Yin XM: Autophagy reduces acute ethanol-induced hepatotoxicity and steatosis in mice. Gastroenterology 2010, 139: $1740-1752$

37. Lanthier N, Starkel P: Treatment of severe alcoholic hepatitis: past, present and future. Eur J Clin Invest 2017, 47:531-539

38. Thursz MR, Richardson P, Allison M, Austin A, Bowers M, Day CP, Downs N, Gleeson D, MacGilchrist A, Grant A, Hood S, Masson S, McCune A, Mellor J, O'Grady J, Patch D, Ratcliffe I, Roderick P, Stanton L, Vergis N, Wright M, Ryder S, Forrest EH; STOPAH Trial: Prednisolone or pentoxifylline for alcoholic hepatitis. N Engl J Med 2015, 372:1619-1628

39. Yang X, Wang H, Ni HM, Xiong A, Wang Z, Sesaki H, Ding WX, Yang L: Inhibition of Drp1 protects against senecionine-induced mitochondria-mediated apoptosis in primary hepatocytes and in mice. Redox Biol 2017, 12:264-273

40. Xu S, Pi H, Chen Y, Zhang N, Guo P, Lu Y, He M, Xie J, Zhong M, Zhang Y, Yu Z, Zhou Z: Cadmium induced Drp1-dependent mitochondrial fragmentation by disturbing calcium homeostasis in its hepatotoxicity. Cell Death Dis 2013, 4:e540

41. Galloway CA, Lee H, Brookes PS, Yoon Y: Decreasing mitochondrial fission alleviates hepatic steatosis in a murine model of nonalcoholic fatty liver disease. Am J Physiol Gastrointest Liver Physiol 2014, 307:G632-G641

42. Yu T, Wang L, Lee H, O'Brien DK, Bronk SF, Gores GJ, Yoon Y: Decreasing mitochondrial fission prevents cholestatic liver injury. J Biol Chem 2014, 289:34074-34088

43. Altamirano J, Miquel R, Katoonizadeh A, Abraldes JG, Duarte-Rojo A, Louvet A, Augustin S, Mookerjee RP, Michelena J, Smyrk TC, Buob D, Leteurtre E, Rincon D, Ruiz P, Garcia-Pagan JC, Guerrero-Marquez C, Jones PD, Barritt AS, Arroyo V, Bruguera M, Banares R, Gines P, Caballeria J, Roskams T, Nevens F, Jalan R, Mathurin P, Shah VH, Bataller R: A histologic scoring system for prognosis of patients with alcoholic hepatitis. Gastroenterology 2014, 146:1231-1239

44. Andrade P, Silva M, Rodrigues S, Lopes J, Lopes S, Macedo G: Alcoholic hepatitis histological score has high accuracy to predict 90day mortality and response to steroids. Dig Liver Dis 2016, 48: 656-660

45. Chedid A, Mendenhall CL, Tosch T, Chen T, Rabin L, Garcia-Pont P, Goldberg SJ, Kiernan T, Seeff LB, Sorrell M: Significance of megamitochondria in alcoholic liver disease. Gastroenterology 1986, 90: $1858-1864$

46. Kim W, Choi Y-I, Joo SK, Jung YJ: Alcoholic hepatitis histological scores predict short-term survival in Asian patients with biopsyproven alcoholic hepatitis. J Hepatol 2017, 66:S118

47. Rakhmatullina D, Ponomareva A, Gazizova N, Minibayeva F: Mitochondrial morphology and dynamics in Triticum aestivum roots in response to rotenone and antimycin A. Protoplasma 2016, 253: $1299-1308$

48. Brooks C, Wei Q, Cho SG, Dong Z: Regulation of mitochondrial dynamics in acute kidney injury in cell culture and rodent models. J Clin Invest 2009, 119:1275-1285

49. Cassidy-Stone A, Chipuk JE, Ingerman E, Song C, Yoo C, Kuwana T, Kurth MJ, Shaw JT, Hinshaw JE, Green DR, Nunnari J: Chemical inhibition of the mitochondrial division dynamin reveals its role in Bax/Bak-dependent mitochondrial outer membrane permeabilization. Dev Cell 2008, 14:193-204

50. Cui M, Ding H, Chen F, Zhao Y, Yang Q, Dong Q: Mdivi-1 protects against ischemic brain injury via elevating extracellular adenosine in a cAMP/CREB-CD39-dependent manner. Mol Neurobiol 2016, 53: $240-253$

51. Grohm J, Kim SW, Mamrak U, Tobaben S, Cassidy-Stone A, Nunnari J, Plesnila N, Culmsee C: Inhibition of Drp1 provides neuroprotection in vitro and in vivo. Cell Death Differ 2012, 19:1446-1458

52. Park SW, Kim KY, Lindsey JD, Dai Y, Heo H, Nguyen DH, Ellisman MH, Weinreb RN, Ju WK: A selective inhibitor of drp1, mdivi-1, increases retinal ganglion cell survival in acute ischemic mouse retina. Invest Ophthalmol Vis Sci 2011, 52:2837-2843

53. Xie N, Wang C, Lian Y, Zhang H, Wu C, Zhang Q: A selective inhibitor of Drp1, mdivi-1, protects against cell death of hippocampal neurons in pilocarpine-induced seizures in rats. Neurosci Lett 2013, 545:64-68

54. Sharp WW, Beiser DG, Fang YH, Han M, Piao L, Varughese J, Archer SL: Inhibition of the mitochondrial fission protein dynaminrelated protein 1 improves survival in a murine cardiac arrest model. Crit Care Med 2015, 43:e38-e47 